\title{
AVALIAÇÃO DA IMPORTÂNCIA DOS EVENTOS DO eduScrum, DOS CANAIS DO MICROSOFT TEAMS, TRELLO E DO JAMBOARD NO ENSINO REMOTO EMERGENCIAL
}

\author{
DOI: 10.37702/2175-957X.COBENGE.2021.3407
}

Norimar de Melo Verticchio - N_verticchio@yahoo.com.br

Ifmg betim

Rua Rio Elba 99

32280-310 - Contagem - MG

Marco Antonio Barbosa Braga - marcobraga@namelab.education CEFETRJ

Rua General Goes Monteiro 08

22290-080 - RIO DE JANEIRO - RJ

Resumo: A suspenção das aulas presenciais devido à pandemia do COVID-19 fez com que as universidades adotassem o Ensino Remoto Emergencial (ERE), que foi autorizado pelo MEC por meio da portaria no 343. Para avaliar o impacto da utilização do eduScrum, do Microsoft Teams, do Trello e Jamboard no desenvolvimento dos trabalhos em grupo durante o ERE, foi realizada uma pesquisa nas disciplinas Fenômenos de Transporte e Processos de Fabricação 2 ministradas nos cursos de Engenharia do IFMG campus Betim. Para isso foi aplicado um questionário no qual $77 \%(n=33)$ dos alunos avaliaram o grau de importância para o trabalho em grupo dos eventos do eduScrum (Formação da equipe, planejamento do sprint, revisão do sprint, retrospectiva do sprint e reuniões diárias) e as ferramentas de tecnologia da informação utilizadas (Microsoft Teams, Trello e Jamboard). Desses alunos 100\% consideraram o Planejamento do Sprint, a Revisão do Sprint e a Retrospectiva do Sprint como sendo Fundamental ou Importante para o desenvolvimento do trabalho em grupo. Com relação às tecnologias utilizas os "canais" do Microsoft Teams foi considerado Fundamental ou Importante para $86,7 \%$ dos respondentes. O ERE trouxe novos desafios aos docentes, sendo assim é importante que novas metodologias e ferramentas que auxiliem o trabalho do docente sejam testadas e validadas.

Palavras-chave: eduScrum, TIC, Ensino Remoto Emergencial, Trabalho em grupo, Scrum 


\section{INTRODUÇÃO}

A Organização Mundial de Saúde (OMS) elevou, no dia 11 de março de 2020, o estado de contaminação de Covid-19 à pandemia (WHO, 2020). Muitas transformações ocorreram na sociedade devido à alta taxa de transmissão do novo Corona vírus. A medida de maior impacto que foi adotada pela maioria dos países foi o isolamento social, gerando assim impactos sociais, econômicos e educacionais imediatos (NETO, 2020). No âmbito educacional brasileiro houve a paralização compulsória de todas as atividades presenciais nas escolas.

Em 17 de março de 2020, o Ministério da Educação (MEC) publicou a portaria nº 343, que dispôs sobre a substituição das aulas presenciais por aulas em meios digitais enquanto durar a situação de pandemia do COVID-19 (BRASIL, 2020).

No IFMG, as aulas presenciais foram suspensas a partir do dia 18 de março de 2020, ficando a cargo de cada campus ou a suspenção do calendário acadêmico ou a continuação das aulas por meio do Ensino Remoto Emergencial (ERE). O campus Betim do IFMG optou pela continuação das atividades acadêmicas por meio do ERE. Todas as disciplinas foram cadastradas no Ambiente Virtual de Aprendizagem (AVA) e os alunos realizaram a sua auto inscrição nas mesmas disciplinas que estavam regularmente matriculados.

$\mathrm{Na}$ disciplina de Mecânica dos Fluidos, do curso de graduação em Engenharia Mecânica do IFMG campus Betim, o ensino presencial estava utilizando pela primeira vez a metodologia PBL junto com o framework eduScrum. Após a suspensão das atividades presenciais, optou-se por manter a utilização dessas metodologias de ensino, realizando apenas as adequações necessárias para o ensino remoto emergencial.

No final do primeiro semestre foi aplicado um questionário do Google Form®, no qual foram realizadas perguntas aos alunos de Mecânica dos Fluidos sobre o ERE, a utilização do eduScrum e o desenvolvimento do trabalho em grupo. A partir das respostas dos alunos pode-se observar que o ERE e as ferramentas utilizadas não favoreceram o trabalho em grupo.

Com o objetivo de melhorar o rendimento do trabalho em grupo desenvolvido dentro da metodologia eduScrum, optou-se por mudar, no segundo semestre de 2020, a plataforma de aulas síncronas, trocando o Google Meet ${ }^{\circledR}$ pelo Microsoft Teams®, além disso foram utilizadas outras ferramentas digitais para o trabalho colaborativo, como o Trello® e o Jamboard®.

O presente trabalho tem como objetivo avaliar o grau de importância dos eventos do eduScrum e das ferramentas de Tecnologia da Informação (TiC) utilizadas no ERE tiveram no desenvolvimento do trabalho em grupo nas disciplinas de Fenômenos de transporte e Processos de Fabricação 2 ministradas no segundo semestre de 2020 nos cursos de Engenharia do campus Betim do IFMG.

\section{EDUSCRUM}

Os pilares do Scrum foram desenvolvidos por Takeuchi e Nonaka (1986) e a sua aplicação no desenvolvimento de softwares foi descrito por Degrace e Stahl (1990). Os princípios, as regras, os papéis, os eventos e os artefatos utilizados, atualmente, no Scrum foram apresentados no documento denominado "Guia Scrum". Nesse documento, o Scrum é definido como:

"[...] um framework estrutural que está sendo usado para gerenciar o desenvolvimento de produtos complexos desde o início de 1990. Scrum não é um processo ou uma técnica para construir 
produtos; em vez disso, é um framework dentro do qual você pode empregar vários processos ou técnicas." (Schwaber \& Sutherland, 2017, p. 3)

Já o eduScrum foi desenvolvido pelo professor de Química do Colégio Ashram, Willy Wijnands, na cidade de Alphen aan den Rijn na Holanda. A palavra eduScrum é formada pela união das palavras Educação e Scrum, ou seja, é uma adaptação do framework Scrum para o ambiente educacional. Baseando-se nos princípios do Scrum, o professor implementou em 2011, essa metodologia em suas turmas com alunos na faixa etária entre 12 e 18 anos (Sutherland, 2016). Para auxiliar outros professores na implantação dessa versão do Scrum adaptado para a educação, Wijnands fundou o eduScrum e produziu o Guia eduScrum (Delhij, van Solingen, \& Wijnands, 2016).

O eduScrum possui três atores básicos muito bem definidos: o Product Owner, o Master Scrum e a equipe de estudantes. Além deles, também existe uma série de eventos que são necessários para que o desenvolvimento da aprendizagem possa acontecer durante o processo.

\subsection{Eventos do eduScrum}

Delhij et al. (2016) e Wijnands e Stolze (2019) descrevem os seguintes eventos do eduScrum: formação das equipes, Definição de Pronto (DoD) e Definição de Diversão (DoF), planejamento do sprint, desenvolvimento do sprint, Reuniões de stand up, Gráfico Run up, revisão do sprint e retrospectiva do sprint. A figura 1 mostra quais etapas são únicas e quais etapas que são recorrentes.

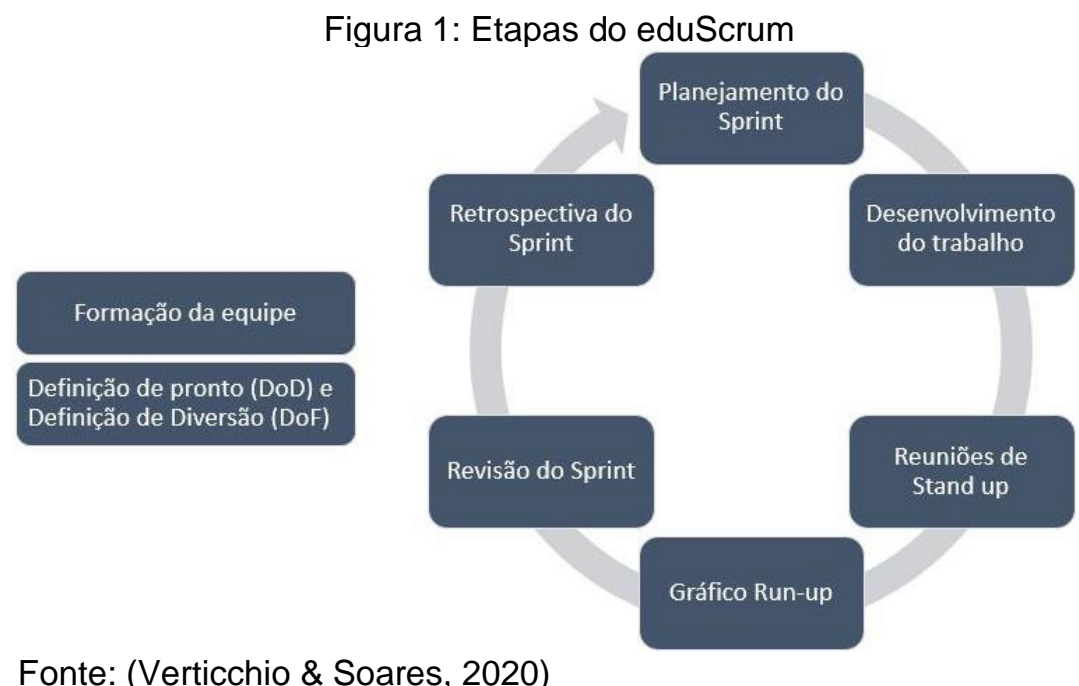

As equipes são formadas considerando as competências e habilidades dos alunos. Também se leva em consideração os conhecimentos anteriores deles. Essa etapa é realizada por meio de um questionário aplicado na primeira aula. Nesse questionário os estudantes selecionam cinco características principais, dentre as quais estão a: liderança, organização, comunicação, projetista, planejador etc. O professor define os líderes das equipes por meio das características selecionadas pelos alunos e esses líderes selecionam os outros alunos por meio das suas características. Desta forma as equipes formadas são multifuncionais e heterogêneas. 
$\mathrm{Na}$ segunda etapa cada equipe discute e apresenta a sua Definição de Pronto que também pode ser denominada de "Definição de Qualidade" ou de aceite (DoD, Definition of Done) e da sua "Definição de Diversão" (DoF, Definition of Fun). Segundo Verticchio e Soares $(2020$, p.6) "esses acordos se referem à qualidade das entregas e como a equipe trabalhará de forma que o desenvolvimento do trabalho ocorra de forma agradável."

Todas as outras etapas ocorrem durante o sprint. Wijnands e Stolze (2019) definem o sprint como sendo um tempo fixo no qual o trabalho é planejado, desenvolvido e avaliado.

No planejamento do sprint, o professor apresenta qual a visão geral da disciplina e do trabalho a ser desenvolvido naquele sprint. O número de aulas que os alunos terão disponíveis para realizar o desenvolvimento do trabalho, as unidades didáticas do plano de ensino que serão contemplados no Sprint, quais os tipos de entregas, qual o modelo de avaliação que será utilizado, quais os critérios de aceitação e, por fim, quais são as datas importantes. A partir dessas informações os alunos planejam todas das tarefas que serão realizadas durante aquele Sprint. Essas tarefas são escritas em notas adesivas (post-it) colocadas no Kanban, na coluna "a fazer", conforme ilustrado na Figura 2.

Figura 2: Modelo de Kanban utilizado durante o eduScrum

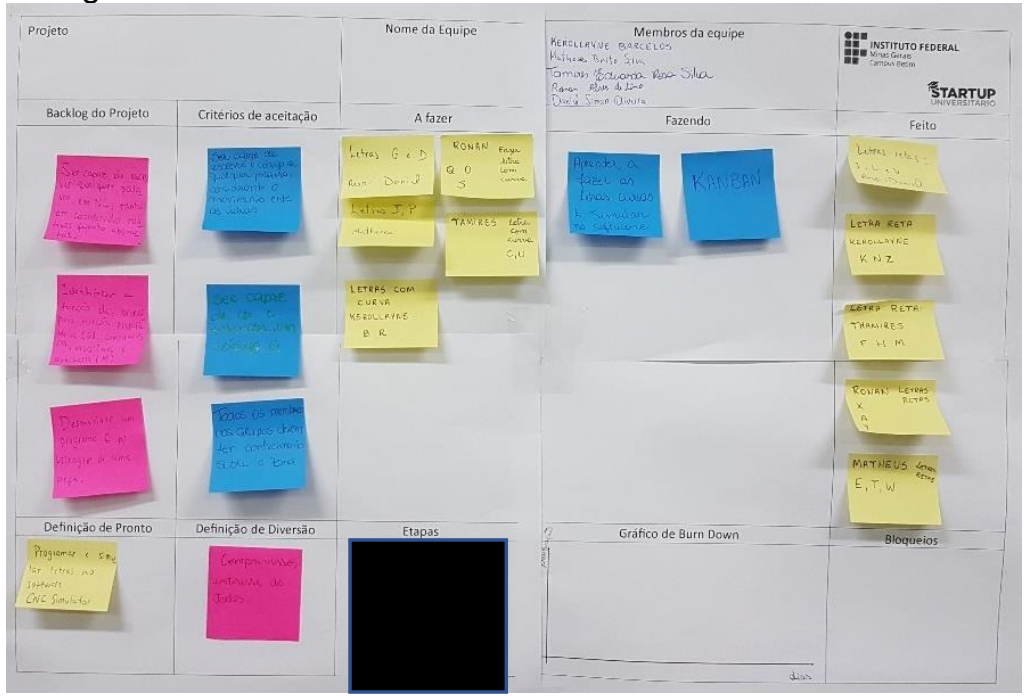

Fonte: Próprio autor

$\mathrm{Na}$ etapa de desenvolvimento do sprint os alunos trabalharão em equipe para cumprir todas as tarefas propostas na etapa de planejamento. Enquanto a equipe estiver realizando a tarefa proposta, as notas adesivas ficam na coluna "fazendo" do Kanban e, após a conclusão da tarefa, a nota adesiva é deslocada para a coluna "feito". Desta forma toda a equipe e o professor conseguem fazer a gestão do trabalho. Somente após a tarefa cumprir os critérios estabelecidos na DoD e nos critérios de aceitação, ela é deslocada para a coluna "feito" do Kanban mostro na Figura 2. Durante o desenvolvimento do trabalho são realizados dois eventos intermediários, as reuniões de Stand-up e a construção do gráfico Run-up ou Burn down.

As reuniões de Stand-up são realizadas no início de cada aula. Participam dessa reunião o Scrum Master e a equipe de estudantes. É recomendável que o professor apenas observe o desenvolvimento dessa reunião. Nessa reunião os alunos se posicionam em frente ao seu Kanban e iniciam a reunião que deve durar no máximo 5 minutos, no qual cada estudante responde às seguintes perguntas:

- O que eu fiz desde a última reunião de Stand-up?

- O que eu vou fazer nessa aula e até a próxima reunião de Stand-up? 
- Quais os obstáculos ou impedimentos estão me atrapalhando ou atrapalhando a equipe?

No final da etapa de desenvolvimento ocorrem as etapas de revisão do sprint e a retrospectiva do sprint. Segundo Verticchio e Soares $(2020$, p.8) "ambas são importantes para o fornecimento de feedback e inspeção do trabalho que foi desenvolvido."

$\mathrm{Na}$ revisão do Sprint os alunos apresentam o que eles desenvolveram no Sprint e recebem o feedback imediato do professor. A maneira como acontecerá a revisão é definida pelo professor, podendo ser uma apresentação oral, a construção de um protótipo, uma redação, a construção de um site, um teste escrito, um TBL, entre outros. Essas entregas são avaliadas e fazem parte do processo formativo dos alunos.

Antes do início do próximo sprint as equipes fazem a retrospectiva do sprint que é uma autoavaliação do trabalho da equipe e de cada um dos alunos. Os objetivos da retrospectiva do Sprint são os seguintes:

- Inspecionar como ocorreu o último Sprint, no que diz respeito a pessoas, relações, processos e ferramentas;

- Identificar as coisas que correram bem e potenciais melhorias que podem ajudar no desenvolvimento do trabalho;

- Criar um plano para implementar melhorias no modo como a equipe realiza o seu trabalho.

Verticchio e Soares (2020) dividem a retrospectiva do Sprint em três partes:

- Os alunos avaliam a forma como a equipe trabalhou, identificando pontos de melhoria.

- Cada aluno avalia os demais membros de sua equipe e realiza uma autoavaliação, levando em consideração a utilização das qualidades identificadas na formação da equipe e propondo melhorias.

- A equipe discute o que eles deveriam parar de fazer para melhorar o seu rendimento.

\section{TRABALHO DE EQUIPE NO ERE}

Com o objetivo de auxiliar os alunos no trabalho em equipe optou-se pela utilização da plataforma Microsoft Teams para a realização das aulas síncronas. O Microsoft Teams, segundo Arrieta et al. (2019), possibilita que o docente trabalhe com grupos de alunos, pois concentra várias ferramentas que auxiliam o trabalho colaborativo, tais como: os canais, calendários, blocos de notas interativos, documentos compartilhados e chats)

Ainda segundo Arriela et al. (2019) o Microsoft Teams pode ser utilizado como um Sistema de Gestão da Aprendizagem, porém no campus Betim optou-se pela utilização do Moodle como sistema de gestão da aprendizagem, deste modo, o Microsoft Teams será utilizado apenas para as aulas síncronas e a gestão do trabalho em grupo.

Ao criar as equipes das disciplinas a plataforma cria um canal chamado de Geral, nesse canal ocorreram as aulas síncronas e as trocas de informações com toda a turma, para o desenvolvimento do trabalho das equipes foi criado um canal distinto para cada grupo, conforme mostra a figura 3. Os canais são espaços de colaboração onde professores e alunos trabalham colaborativamente nas tarefas ou acessam o material de estudo. (MICROSOFT, 2018) 
Figura 3: Canais utilizados na disciplina de Fenômenos de Transporte

\begin{tabular}{|l|} 
Fenômenos de Transporte - ... $\cdots$ \\
Geral \\
Grupo 1 - FT \\
Grupo 2 - FT \\
Grupo 3 - FT \\
Grupo 4 - FT \\
Grupo 5 - FT \\
Reunião individual \\
\hline
\end{tabular}

Fonte: Próprio autor

No canal específico de cada grupo os alunos realizaram reuniões ao vivo durante a aula síncrona, possibilitando assim que interagissem e colaborassem nos seguintes eventos: planejamento do sprint, desenvolvimento do sprint e retrospectiva do sprint.

Outra vantagem da utilização dos Canais é que o professor pode ser chamado pelo grupo por meio da ferramenta de "menção" do chat, ou seja, ao utilizar @nome do professor no chat, o professor é notificado e pode acessar e participar da reunião daquele grupo.

Para auxiliar nos eventos de planejamento do sprint e desenvolvimento do sprint e substituir o Kanban físico, optou-se pela utilização do Trello® que é um programa de Gestão de projetos no qual utiliza um sistema visual de etiquetas que possuem informações sobre as tarefas que precisam ser executados para cumprir um objetivo específico.

Para padronizar e facilitar a utilização do Trello o professor criou um quadro para cada grupo e realizou o sincronismo do mesmo no canal de cada equipe, conforme mostra a figura 3, desta forma toda a gestão do grupo pode ser realizada dentro do Microsoft Teams.

Figura 3: Utilização do Trello dentro do Microsoft Teams.

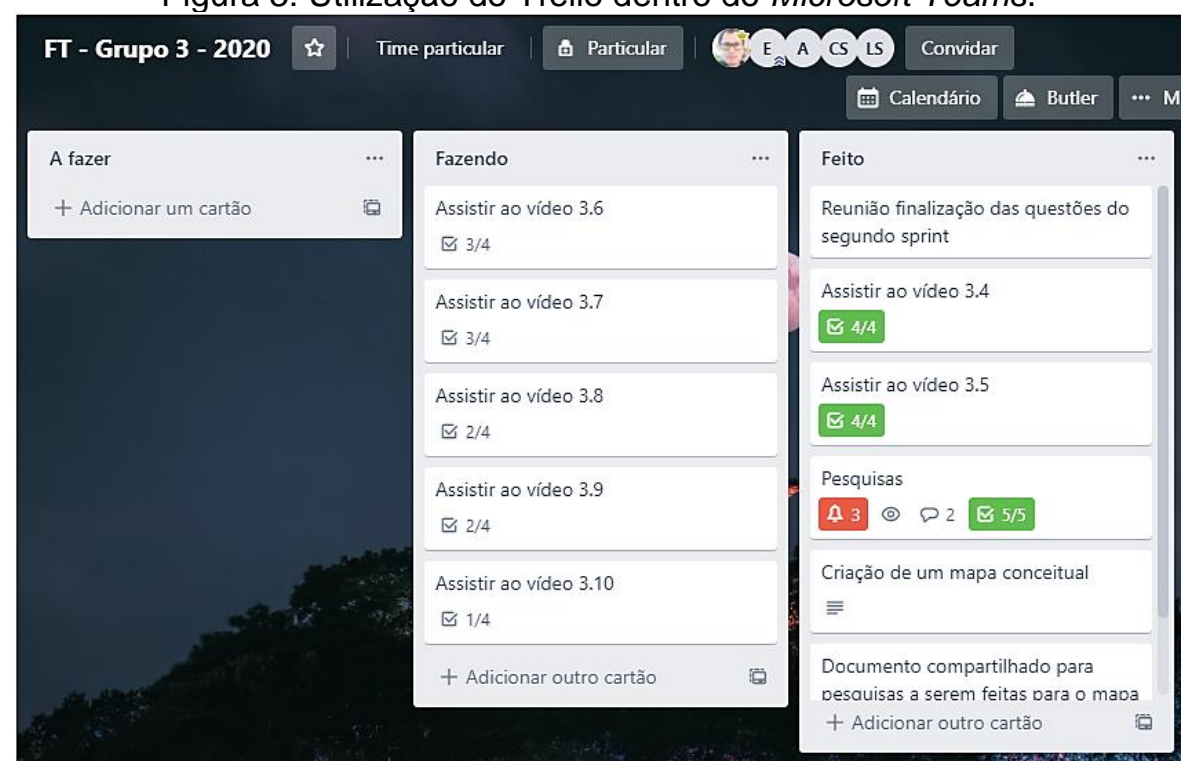

Fonte: Próprio Autor

Outra ferramenta utilizada para auxiliar os alunos na realização da retrospectiva do sprint foi o Jamboard® que é uma tela interativa desenvolvida pelo Google® no qual é possível utilizar notas adesivas virtuais para realizar trabalhos colaborativos. Diferente do 
Trello que tem como objetivo a gestão de processos e projetos o Jamboard é um quadro de interação, ou seja, os alunos criam e discutem de forma colaborativa. A figura 4 apresenta o jamboard utilizado na retrospectiva do sprint.

Figura 4: Jamboard utilizado na retrospectiva do sprint.

0 que foi bem
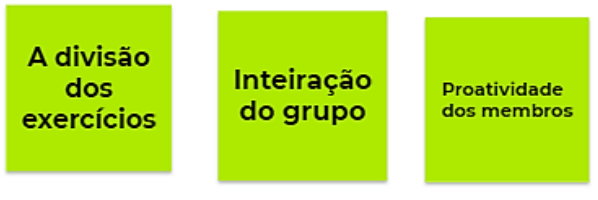

Fonte: Próprio Autor
$\mathrm{O}$ que pode melhorar
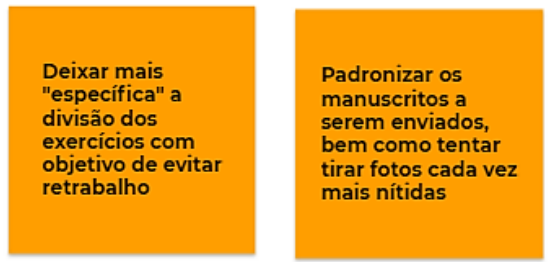

\section{AVALIAÇÃO DAS FERRAMENTAS E EVENTOS DO EDUSCRUM}

Para avaliar o impacto das ferramentas utilizadas e dos eventos do eduScrum foi aplicado um questionário, que foi respondido por $71 \%$ dos alunos das disciplinas de Fenômenos de Transporte e Processo de Fabricação 2. Foi questionado aos alunos qual o grau de importância das ferramentas utilizadas e dos eventos realizados. A tabela 1 apresenta os resultados obtidos.

Foi utilizado uma escala de likert, no qual os alunos avaliaram cada um dos eventos e ferramentas o grau de importância por meio das seguintes opções: "Fundamental", "Muito Importante", "Importante", "Sem Importância" e "Desnecessária". Para realizar a análise, essas respostas foram agrupadas da seguinte forma: No grupo "Fundamental" estão somadas as respostas "Fundamental" e "Muito Importante" e no grupo "Sem Importância" estão agrupadas as respostas "Sem Importância" e "Desnecessária".

Tabela 1: Importância dos eventos eduScrum e ferramentas utilizadas

\begin{tabular}{lccc}
\hline Eventos e ferramentas & Fundamental & Importante & Sem importância \\
\hline Grupos por competência & $53.3 \%$ & $40.0 \%$ & $6.7 \%$ \\
\hline Manutenção do mesmo grupo & $60.0 \%$ & $36.7 \%$ & $3.3 \%$ \\
\hline Utilização do eduScrum & $20.0 \%$ & $63.3 \%$ & $16.7 \%$ \\
\hline Planejamento do Sprint & $56.7 \%$ & $43.3 \%$ & $0.0 \%$ \\
\hline Revisão do Sprint & $66.7 \%$ & $33.3 \%$ & $0.0 \%$ \\
\hline Retrospectiva do Sprint & $46.7 \%$ & $53.3 \%$ & $0.0 \%$ \\
\hline Reuniões diárias & $30.0 \%$ & $53.3 \%$ & $16.7 \%$ \\
\hline Uso do Jamboard & $0.0 \%$ & $53.3 \%$ & $46.7 \%$ \\
\hline Uso dos canais do Teams & $66.7 \%$ & $20.0 \%$ & $13.3 \%$ \\
\hline Uso do Trello & $30.0 \%$ & $60.0 \%$ & $10.0 \%$ \\
\hline Fonte: Próprio Autor & & &
\end{tabular}

Observa-se na tabela 1, que os eventos planejamento do sprint, revisão do sprint e retrospectiva do sprint foram considerados ou "Fundamentais" ou "Importantes" para todos os respondentes, destacando-se que a revisão do sprint foi considerado "Fundamental" para $66,7 \%$ dos alunos.

Com relação as ferramentas utilizadas verificam-se que os canais do Microsoft Teams foram considerados por $66,7 \%$ dos alunos como sendo "Fundamentais" para o 
trabalho em grupo, já o Trello foi considerado "Fundamental" para $30 \%$ dos alunos, porém apenas $10 \%$ dos alunos consideraram a sua utilização como sendo "Sem importância". Nenhum aluno considerou o Jamboard como sendo "Fundamental".

A formação dos grupos por competência e a manutenção do mesmo grupo também foram considerados, respectivamente, por $93,3 \%$ e $96,7 \%$ dos alunos como sendo "Fundamentais" ou "Importantes" para o desenvolvimento do trabalho em grupo.

\section{CONCLUSÃO}

Nesse trabalho buscou-se avaliar o grau de importância na perspectiva dos alunos das ferramentas e metodologias utilizadas nas disciplinas de Fenômenos de transporte e Processos de fabricação 2 nos cursos de Engenharia do IFMG.

O Ensino Remoto Emergência trouxe novos desafios tanto para os professores quanto para os alunos. Alguns desses desafios que se pode destacar são: Como avaliar o aprendizado dos estudantes, como garantir acesso, como avaliar o nível de participação, como desenvolver e trabalhar em grupo.

Para melhorar e possibilitar o desenvolvimento de trabalhos em grupo ou equipes foi utilizado o framework eduScrum e várias ferramentas de Tecnologia da informação e comunicação (TIC), tais como, canais no Microsoft Teams, os quadros do Trello e o jamboard.

Segundo os alunos o Planejamento, a Revisão e a Retrospectiva do Sprint foram eventos fundamentais para o desenvolvimento do trabalho em grupo. Com relação as ferramentas de TIC os alunos consideraram a utilização dos canais do Trello como sendo a ferramenta mais importante nesse processo.

Apesar de nenhuma ferramenta tecnologia substituir a interação social que ocorre durante as aulas presenciais, esse trabalho apresenta um caminho alternativo para amenizar a falta de interação entre os alunos que ocorre no Ensino Remoto Emergência.

\section{REFERÊNCIAS}

ARRIETA, Mauricio et al. Convergencia de procesos de docencia universitaria: El uso de la aplicación Teams de Microsoft.

BRASIL. Portaria no 343, de 17 de março de 2020. Diário Oficial da União, Brasília, DF, 18 mar. 2020. Ed. 53. Seção 1, p. 39. Disponível em: https://bit.ly/3k1HLs4. Acesso em: 19 jul. 2020.

DEGRACE, Peter; STAHL, Leslie Hulet. Wicked problems, righteous solutions: a catalogue of modern Software Engineering paradigms. Upper Saddle River: Prentice Hall, 1990.

DELHIJ, Arno.; VAN SOLINGEN, Rini; WIJNANDS, Willy. O guia eduScrum. 2016. Disponível em: https://bit.ly/2SATf9Q. Acesso em: 15 jul. 2020.

MICROSOFT. Welcome to Microsoft Teams. 2018 Disponível em: https://bit.ly/2SwrC1o. Acesso em: 07 out. 2020 
NETO, Joaquim Antunes. Sobre ensino, aprendizagem e a sociedade da tecnologia: por que se refletir em tempo de pandemia?. Prospectus, v. 2, n. 1, p. 28-38, 2020.

SCHWABER, Ken.; SUTHERLAND, Jeff. The Scrum guide. The definitive guide to Scrum: The rules of the game, 2017.

SUTHERLAND, Jeff. Scrum: a arte de fazer o dobro do trabalho na metade do tempo. Rio de Janeiro (Brasil): Leya, 2016.

TAKEUCHI, Nonaka; NONAKA, Ikujiro. The new product development game. Harvard Business Review, 64(1), 137-146, 1986.

VERTICCHIO, Norimar de Melo.; SOARES, Gabriel de Oliveira. Perception of students of an integrated technical course in industrial automation on the use of eduScrum. Research, Society and Development, v. 9, n. 7, 2020.

WIJNANDS, Willy.; STOLZE, Alisa. Transforming Education with eduScrum. In: PARSONS, D.; MACCALLUM, K. (ed.). Agile and lean concepts for teaching and learning. Singapore: Springer, p. 95-114, 2019.

WORLD HEALTH ORGANIZATION (2020). Coronavirus disease 2019 (COVID-19): Situation Report 51. WHO. Disponível em: https://bit.ly/2CXmfEv. Acesso em: 20 jul. 2020.

\title{
IMPORTANCE OF EDUSCRUM EVENTS AND MICROSOFT TEAMS CHANNELS IN EMERGENCY REMOTE EDUCATION
}

\begin{abstract}
The suspension of face-to-face classes due to the COVID-19 pandemic led universities to adopt Emergency Remote Education (ERE), which was authorized by MEC through ordinance No. 343. To assess the impact of using eduScrum, Microsoft Teams, from Trello and Jamboard in the development of group work during the ERE, a research was carried out in the disciplines of Transport Phenomena and Manufacturing Processes 2 given in the Engineering courses of the IFMG Betim campus. For this, a questionnaire was applied in which $77 \%(n=33)$ of the students evaluated the degree of importance for the group work of eduScrum events (Team building, sprint planning, sprint review, sprint retrospective and daily meetings) and the information technology tools used (Microsoft Teams, Trello and Jamboard). Of these students 100\% considered Sprint Planning, Sprint Review and Sprint Retrospective to be Fundamental or Important for the development of group work. Regarding the technologies used, the Microsoft Teams "channels" were considered Fundamental or Important for $86.7 \%$ of the respondents. The ERE brought new challenges to teachers, so it is important that new methodologies and tools that assist the work of the teacher are tested and validated.
\end{abstract}

Keywords: eduScrum, ICT, Emergency Remote Teaching, Teamwork. 